\title{
EFFECTIVE REDIRECTING OF THE MOBILE RoBOT IN A MESSED ENVIRONMENT BASEd ON THE FUZZY LOGIC
}

\author{
Hamed Khosravi and Seyed Ghorshi \\ School of Science and Engineering, Sharif University of Technology, \\ International Campus, Kish Island, Iran.
}

\begin{abstract}
The use of fuzzy logic in redirecting mobile robot is based on two sets of received information. First set is the instantaneous distance of the robot from the obstacle and second set is the instantaneous information of the robot's position. For this purpose, the fuzzy rules base consists of forty-two bases, which is extracted based on the robot's distance from obstacles, and the target position relative to the instantaneous orientation of the robot. In the structure of fuzzy systems, minimal inference engine are considered. Also, Extended Kalman filter is used for localization in a noisy environment. Accordingly, the inputs of the fuzzy systems are determined based on the estimation of the localization process, the information of the obstacles center and the target position. Also, the linear acceleration and instantaneous orientation of the mobile robot are determined by the desired fuzzy structures which are applied to its kinematic model.
\end{abstract}

\section{KEYWORDS}

Mobile robot, Fuzzy logic, Effective redirecting

\section{INTRODUCTION}

A Mobile robot is a robot that are capable of moving and consists of many robots. For the purpose of moving a mobile robot needs a completely coordinated capability, such as motion planning. In motion planning, the goal is a safe motion that will be achievable for the robot. Since a mobile robot works in a real world with static and dynamic properties, one of the most important researches in the robotics is to provide the ability to plan the path and move the mobile robot autonomously. Also for mobile robots, the most important challenges are the operations in uncertain and messy environments along with uncertainties in the model in this field. Various methods have been developed for the accurate robot redirecting in these conditions, which can be divided into three general categories: model-based methods, fuzzy logic-based methods, and neural network-based methods. Model-based methods can be based on a precise model of the environment, plan a route without dealing with obstacles for a mobile robot between the initial points and the target. These methods cannot be used in variable and unknown environments. The main characteristic of data-based methods such as fuzzy logic and neural network is their high response speed and consistency versus measurement noise. These methods are able to routing the sensed data for a mobile robot in real time in a variable and unknown environment. Fuzzy logic and neural networks are powerful tools for controlling complex systems in known or unknown environments. In fuzzy methods, the robot's response is based on the logic of qualitative behaviors in avoiding the obstacle and achieving the target's position [1-3]. In neural methods, the robot response is obtained by predicting a training neural network based on a robot behavior descriptor database. 
International Journal of Fuzzy Logic Systems (IJFLS) Vol.8, No.3, July 2018.

In this paper, the specific features of fuzzy logic will be used to solve the routing problem. These features can be used for planning robot paths in crowded environments. Also, due to the complexity of modeling a nonlinear and variable system, the complexity of modeling its interaction with the environment, and also the advantages of fuzzy controller properties, this type of controller could be a suitable option for controlling the robot's motion. In redirecting the mobile robot, the use of fuzzy logic is based on the two sets of received information. First set is the instantaneous distance of a robot from the obstacles present in the environment, and second is the instantaneous information of the robot's position in Cartesian space. Therefore, as a rule, the inputs of the fuzzy system include the robot's distance from obstacles and the redirection angle of the robot, that is, the angle between the direction of the robot's motion and the target position. On the other hand, according to the decision logic, the output of fuzzy systems can only be linear and rotational accelerations of wheels, robot orientation angle and linear acceleration, the linear and rotational speeds of the mobile robot, etc [4-6].

In developing a fuzzy system, the most important issue is to have a rich fuzzy rule base so that it is the full descriptive of the redirecting issue of the robot in the presence of obstacles in the workspace of the mobile robot. The fuzzy inferences should be made in such a way that the robot is close to its target position with good precision and a fairly high speed of operation and without encountering any of the obstacles. Also, the type of fuzzy inference engine plays an important role in the quality of function of automation redirecting system based on fuzzy logic. Typically, two types of minimum and Mamdani's multiplication are used for the fuzzy inference engine. On the other hand, the center average defuzzification is used to extract fuzzy model. Another important issue in redirecting a mobile robot is to have the instantaneous position of the mobile robot in the Cartesian space. For this purpose, the appropriate sensors should be used. The main problem of using the sensors is the associated noise that decreases the operation of the automatic redirecting system of mobile robot. Therefore, an appropriate algorithm such as extended Kalman filter is useful for accurate estimation of instantaneous position of mobile robot in the presence of noise.

\section{RESEARCH BACKGROUND}

Zhang et al. [7] used the combination of fuzzy logic with RFID technology to determine the instantaneous position of the mobile robot based on the diagnosis of current distance of robot to target position. Fuzzy logic inputs include the speed of the robot and the power index of the received signal and the output is the robot distance to the target position. In order to reduce unpredictable errors due to RFID signal fluctuations, the average strength of current signal and the latest RSSI information are used as the input of the fuzzy system. The extended Kalman filter based on a fuzzy neural network has been proposed in [8] and [9] to improve the localization of a mobile robot in an uncertain environment. The proposed approach is a combination of the Kalman filter installed on a mobile robot with differential setup and covariance matrix matching program of the process and the measurement noise. The goal of this approach is to overcome the divergence of the Kalman filter algorithm in the case of the incorrect selection of covariance matrices. The fuzzy system uses the difference vector between the actual and predicted measurements as the reference factor for setting covariance matrices of the process and the measurement noise. The fuzzy logic that is used to match these matrices determines its membership functions by multilayer feed-forward neural network. Rigatos [10] has used extended Kalman filter and particle filter algorithms for the mobile robot localization. In the proposed method it is assumed that the process and measurement noises obey a Gaussian distribution. This presentation includes the formulation of a linear Kalman filter for continuousdiscrete state and an extension of them for nonlinear systems. Also, the Monte-Carlo method has been used for the formulation and the particle filter is used in the localization of the mobile robot and assuming that the statistical distribution of noise is non-Gaussian. The particle filters are 
used for state estimation systems that there is no clear answer for them. The major weakness of this algorithm is that after a certain number of repetitions, almost all weights of the algorithm will be equal to zero. To avoid this problem, the particles that lead to zeroing the weights become weaker in favor of other particles. Lin and et al. [11] used a hardware configuration based on a few ultrasonic sensors to localize the robot. The configuration has two ultrasound transmitters and three receivers. As the robot moves in the effective range of ultrasonic emission, the estimation of robot's dynamic position is possible using the information of ultrasonic sensors. To update the robot's position, the extended Kalman filter based on fuzzy logic is used. The goal of fuzzy logic is to improve the accuracy of localizing and increasing the consistency of the localization algorithm relative to measurement noise variations or modeling errors. Fuzzy logic automatically performs exponential weighting for covariance matrices of noise of process and measurements. The covariance matrices of noise of process and measurements consist of an exponential term as a weighting factor of near to one. The inputs of the fuzzy system are the mean, variance, and standard deviation of the innovation vector and the output of the weighting system of the weighting factor of the covariance matrices of noise of process and measurement. Input membership functions have been considered as trapezoidal. The ultrasonic localization systems can be divided into two groups: The first group, known as flight time methods, determines space position of the robot by transmission of information through multiple ultrasound sensors from the robot to the surrounding environment. The second group, known as the Active Aperture Position method, has one or more transmitters that operate as an observation tower, and has multiple receivers positioned in predetermined positions on the robot. In general, localization techniques based on the first method do not have high accuracy and flexibility, while the latter is highly accurate. Begum et al. [12] used a combination of fuzzy logic and genetic algorithms to solve the problem of mapping and instantaneous localization of the mobile robot. Accordingly, an island model based on the genetic algorithm will search the most suitable mapping to obtain the best robot localization information. The previous information about the position of the robot is used to accelerate the convergence of the genetic algorithm. Fuzzy logic provides sample based prediction from the current position of the robot by inferring the amount of uncertainty in the position of the robot. This sample collection is applied to the genetic algorithm as a primitive population in order to search for proper mapping with sufficient information. Among the features of the proposed algorithm are: 1) Smart combination of two soft computing methods for the SLAM problem. 2) Providing a fuzzy framework for modeling the distance movement of mobile robot. 3) Introducing a sample-based new approach for mapping and instantaneous positioning of robot. Najjaran et al. [13] examined the real-time path design issue, which includes mapping and designing the motion path of the mobile robot. Also, a special detector is used to scan the robot motion time. The mapping process is done by utilizing the information of laser and ultrasound sensors, and the path design algorithm uses the mapping to define an obstacle-independent path for the detector. Creating a map includes a combination of information and dealing with uncertainties measurement of sensors. The combination of related information uses the hierarchical filtering process to update the real-time map and optimize the scanning process of the robot motion. The filtering process involves a fuzzy adaptive extended Kalman filter, in which the filter gain is matched using a fuzzy model describes the time of robot motion. Demirli et al. [14] proposed a new approach based on fuzzy logic for dynamically localization a mobile robot equipped with several sonar sensors. In this approach, the angular uncertainty and radial inaccuracy of sonar sensors are modeled using probability distributions. Based on sensor information, a local fuzzy composite mapping is constructed which is fitted with a comprehensive mapping from the environment to identify the position of the robot. As a result, a unique fuzzy position or multiple fuzzy candidate positions are obtained. To reduce the number of candidate positions, the robot moves to a new position and a new fuzzy compound mapping is determined. 
International Journal of Fuzzy Logic Systems (IJFLS) Vol.8, No.3, July 2018.

\section{Proposed Method}

\subsection{Redirecting Robot Based on Fuzzy Logic}

When a mobile robot moves in an unknown environment toward the target's position, the motion path independent of encountering obstacles is achieved through the correct response of the mobile robot to the information received from the sensors. To this end, prior knowledge can be used to create the appropriate behavioral patterns for the mobile robot. During the movement of the mobile robot, it is necessary to make a compromise between avoiding the obstacle and moving correctly towards the target based on the information received from the environment. Since the purpose of robot is finding the optimal position without encountering obstacles, the robot's redirecting strategy is designed in two ways: the presence or absence of obstacles. In the absence of an obstacle, the robot redirected towards the target and accelerates relative to target orientation. In the presence of obstacles, the robot decides to orient and accelerate appropriately based on information received from the distance between its instantaneous position and the obstacles around it. When the robot is very close to the obstacle, reduces its speed and takes direction. In general, the control system of redirecting robot is designed to achieve two control commands. The first is to achieve the proper acceleration and the other appropriate directing command. Accordingly, two fuzzy systems are designed separately. In the first fuzzy system, the instantaneous orientation of the robot is executed, and in the second fuzzy system, the linear acceleration of the robot is executed. Therefore, the outputs of each of the two fuzzy systems are as follows:

- $\theta(t)$ : Robot instantaneous orientation

- $a_{L}(t)$ : instantaneous linear acceleration of the robot

Given the presence of obstacles in the robot's work space and the issue of access to the target position, the inputs of both fuzzy systems are as follows:

- $d_{L}$ : The distance of the mobile robot from the left obstacle

- $d_{R}$ : The distance of the mobile robot from the right obstacle

- $d_{C}$ : The distance of the mobile robot from the direct obstacle

- $d_{B}, d_{Y}, d_{H}$ : The distance of the mobile robot from various obstacles

- $\theta_{r}$ : Target orientation

If instantaneous position of mobile robot indicated with $X$ and obstacle center position with $N$, the robot distance to obstacle is equal to $d=(X-N)^{T}(X-N)$. Also, if target position is equal to $X_{d}=\left(x_{d}, y_{d}\right)$, target orientation is equal to $\tan ^{-1}\left(\frac{x-x_{d}}{y-y_{d}}\right)$. The target orientation represents the angle between the direction of robot's motion and the target connector link and the center of the robot. In Figure 1, each of the input and output components of the fuzzy systems is observed based on Cartesian coordinates of the mobile robot and obstacles. In Figure 1, for input variables $d_{L}, d_{R}, d_{C}, d_{B}, d_{V}$, and $d_{H}$, the Near and Far linguistic variables are considered. The Target orientation $\theta_{r}$ includes five linguistic variables such as big negative (NB), small negative $(\mathrm{NS})$, zero $(\mathrm{Z})$, small positive (PS), and big positive (PB). Also, output variable $\theta(t)$ presents five linguistic variables such as relative big negative (RNB), relative small negative (RNS), zero $(\mathrm{Z})$, relative small positive (RPS) and relative big positive (RPB). The output variable $a_{L}(t)$ includes three output variables such as decreasing acceleration (DECE), zero acceleration (ZACC), and increased acceleration (ACCE). In Figure 2, the structure of fuzzy systems has been presented based on the information received from the distance of the mobile robot to the center of the obstacles and the target orientation. In the proposed structure, the characteristics of the fuzzy system are as follows: Single fuzzifier, center average defuzzification, and minimal inference 
engine. According to the structure of the fuzzy system in Figure 2, the inputs include the robot's distance from the left, right, and opposite obstacles, and the target instantaneous

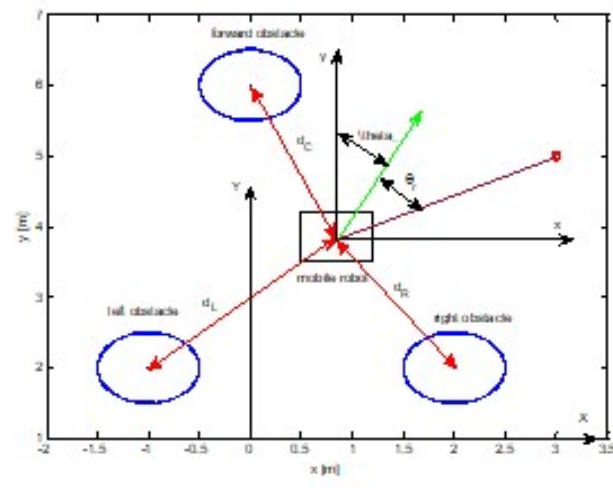

Figure 1. Input components of fuzzy systems based on the instantaneous position of the robot and the position of the obstacles.

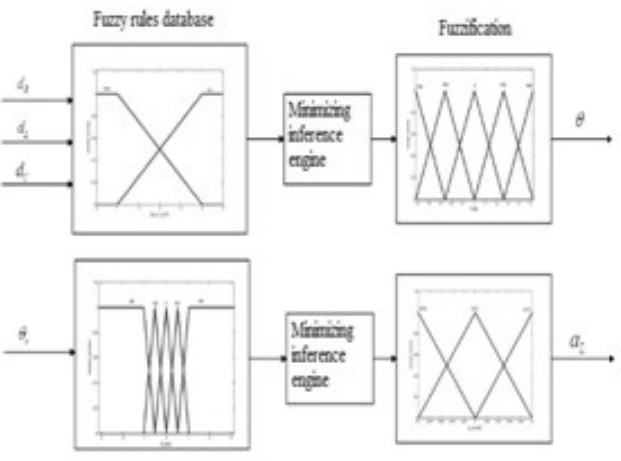

Figure 2. The structure of fuzzy systems for redirecting of the parallel robot.

orientation that enters its associated membership functions based on the fuzzy rules base. Then, the inference is performed for each fuzzy rule based on the minimal inference engine and also the fuzzy rules base. Finally, the orientation and linear acceleration outputs of the robot are determined based on center average defuzzification.

In order to form the base of the fuzzy rules, one should pay attention to the following two issues:

1. When there is no obstacle in the robot's work space or the robot's distance is high, the reactive behavior of the mobile robot is determined by the obstacle position [15-22]. In this situation, the robot accelerates and gradually moves towards the target. Therefore, based on five possible target orientations, five fuzzy rules are considered for behavior of the robot:

- The robot accelerates if the robot's distance is far from the left obstacle, the robot's distance is far from the right obstacle, the robot's distance is far from the direct obstacle and the target orientation is big negative, small negative, zero, small positive and large positive.

- The direction of robot is big negative if the robot's distance is far from the left obstacle, robot's distance is far from the right obstacle, the robot's distance is far from the direct obstacle, and the target orientation is big negative.

- The direction of robot is small negative if the robot's distance is far from the left obstacle, the robot's distance is far from the right obstacle, the robot's distance is far from the direct obstacle, and the target orientation is small negative.

- The direction of robot is zero if the robot's distance is far from the left obstacle, the robot's distance is far from the right obstacle, the robot's distance is far from the direct obstacle, and the target orientation is zero.

- The direction of robot is small positive if the robot's distance is far from the left obstacle, the robot's distance is far from the right obstacle, the robot's distance is far from the direct obstacle, and the target orientation is small positive.

- The direction of robot is big positive if the robot's distance is far from the left obstacle, the robot's distance is far from the right obstacle, the robot's distance is far from the direct obstacle, and the target orientation is big positive. 
International Journal of Fuzzy Logic Systems (IJFLS) Vol.8, No.3, July 2018.

2. When there is an obstacle in the robot's work space or there are low distance between the robot and obstacle, in this situation, the robot decelerates and its orientation is determined by the target position relative to the orientation of the robot. In these situations the fuzzy rules can be written as:

- If the target is on the side in line with the direction of the robot rotation, the robot orientation is relatively small negative and small positive.

- When the distance of the mobile robot is low from the left, right, and direct obstacles, and the rotation of the robot is high, the robot acceleration decreases.

Regarding the structure of fuzzy systems, if the membership functions of inputs $d_{L}, d_{R}, d_{C}, d_{B}, d_{V}, d_{H}$ and $\theta_{r}$ have been indicated by $\mu_{d_{L}}^{l}, \mu_{d_{R}}^{l}, \mu_{d_{C}}^{l}, \mu_{d_{B}}^{l}, \mu_{d_{V}}^{l}, \mu_{d_{H}}^{l}, \mu_{\theta_{r}}^{l}$ respectively and the outputs-membership functions center of $\theta$ and $a_{L}$ have been indicated by $w_{\theta}^{l}$ and $w_{a_{L}}^{l}$. Then, based on single fuzzifier, center average defuzzification, and minimal inference engine, relationship between inputs and outputs of fuzzy systems will be represented as

$$
\begin{aligned}
& \theta(t)=\frac{\sum_{l=1}^{M} w_{\theta}^{l} \min \left\{\mu_{d_{L}}^{l}, \mu_{d_{R}}^{l}, \mu_{d^{\prime}}^{l}, \mu_{d_{B}}^{l}, \mu_{d_{V}}^{l}, \mu_{d_{H^{\prime}}}^{l}, \mu_{\theta_{r}}^{l}\right\}}{\sum_{l=1}^{M} \min \left\{\mu_{d_{L}}^{l}, \mu_{d_{R},}^{l}, \mu_{d_{C^{\prime}}}^{l}, \mu_{d_{B}}^{l}, \mu_{d_{V}}^{l}, \mu_{\left.d_{H^{\prime}}, \mu_{\theta_{r}}^{l}\right\}}^{l}\right\}} \\
& a_{L}(t)=\frac{\sum_{l=1}^{M} w_{a_{L}}^{l} \min \left\{\mu_{d_{L^{\prime}},}^{l} \mu_{d_{R},}^{l}, \mu_{d^{\prime}}^{l}, \mu_{d_{B},}^{l}, \mu_{d_{V}}^{l}, \mu_{d_{H^{\prime}}}^{l}, \mu_{\theta_{r}}^{l}\right\}}{\sum_{l=1}^{M} \min \left\{\mu_{d_{L}{ }^{\prime}}^{l} \mu_{d_{R}}^{l}, \mu_{d_{C^{\prime}},}^{l} \mu_{d_{B},}^{l}, \mu_{d_{V^{\prime}}}^{l}, \mu_{\left.d_{H^{\prime}}, \mu_{\theta_{r}}^{l}\right\}}^{l}\right\}}
\end{aligned}
$$

\subsection{Assessing the Redirecting System of Robot Based on Fuzzy Logic}

To assess the performance of proposed fuzzy logic for redirecting robot in the presence of obstacles in its work space, the kinematic model of robot is considered as follow.

$$
\begin{aligned}
& \dot{x}_{Q}=v_{1} \cos \emptyset \\
& \dot{y}_{Q}=v_{1} \sin \emptyset \\
& \dot{\emptyset}_{Q}=\frac{1}{D} v_{1} \tan \emptyset \\
& \dot{\psi}_{Q}=v_{2}
\end{aligned}
$$

where $\mathrm{v}_{1}$ and $\mathrm{v}_{2}$ are indicators of linear speed and rotation speed of rear wheels, respectively. In other words, these represent driving speed and steering speed, respectively. The initial position of the robot is always assumed in the source. In the robot work space, it is assumed that there are six circular obstacles with a radius of 0.5 meter. It is initially assumed that the robot's target position is at the $x^{*}=(-0.5,7)$ point. The stopping condition of the robot is to achieve a distance of less than 0.01 meter from the target. In Figure 3, the motion of the mobile robot is observed from the source to the target position. Based on Figure 3, the fuzzy logic could appropriately redirect robot toward desired position and, at the same time, avoiding encountered obstacles. There are two issues that need to be addressed in redirecting the mobile robot.

First: Since the exact selection of the membership function center of outputs of fuzzy system requires detailed information about the behavioral nature of the mobile robot and the robot's work space, two correction factors are used one for robot orientation and another for linear acceleration. In relation to the first target position, the orientation correction coefficient is considered 0.65 and the linear acceleration correction coefficient is 0.35 . 
International Journal of Fuzzy Logic Systems (IJFLS) Vol.8, No.3, July 2018.

Second: Different selection of the orientation membership function center causes the mobile robot moves in a different ways toward its target. Based on Figure 4, the mobile robot's distance to the target gradually decreases and reaches to zero after approximately 9100 repetitions.

The values of linear and rotational speeds of the rear wheels are presented in Figure 5. It can be seen that the linear velocity has steadily decreasing behavior, while the velocity has larger angular variation and larger range at some moments. Also, the tracking error in lines $X$ and $Y$ is

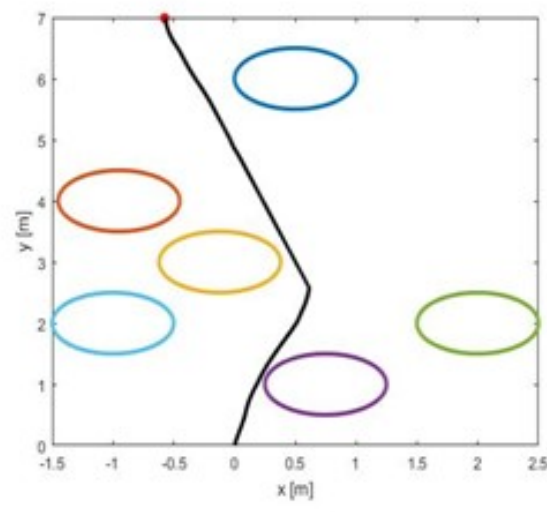

Figure 3. Movement path of the mobile robot from the first target position
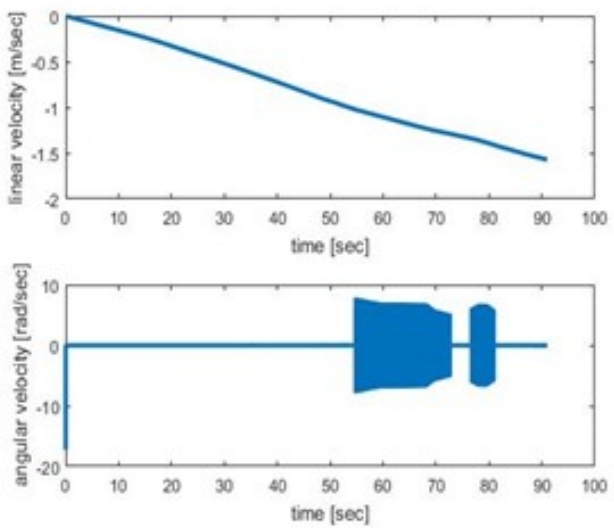

Figure 5. Linear and angular velocity of the tracking the first target position.

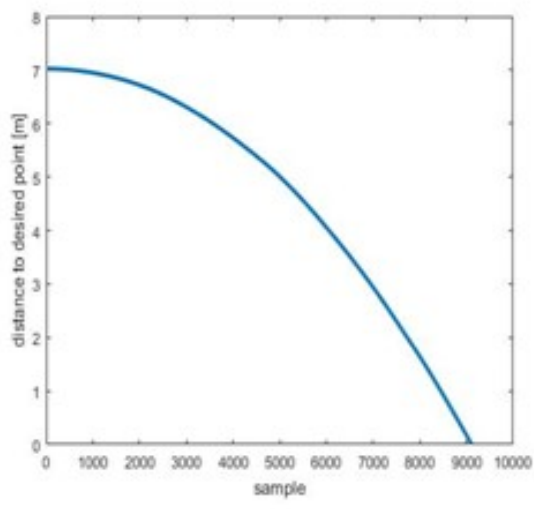

Figure 4. Instantaneous distance of the mobile robot to the first target with fuzzy logic.
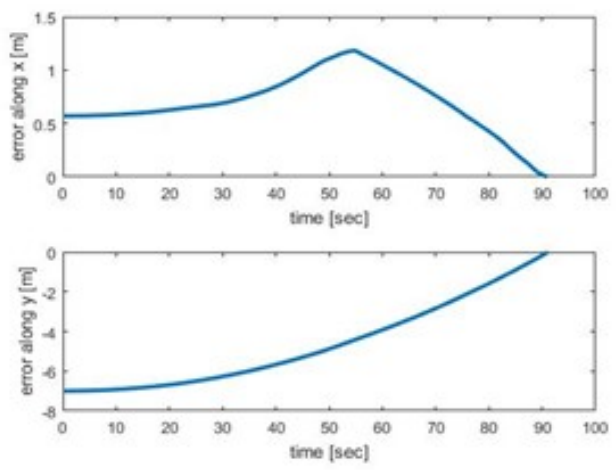

Figure 6. Tracing error of mobile robot in mobile robot in direction of $X$ and $Y$ in tracking the first target position

closed to zero after about 91 seconds according to the Figure 6 . In the following experiment, it is assumed that target situation is $(0,8)$. The initial position of robot is in source. Also, the correction coefficient of center of orientation membership functions and linear velocity are considered 0.67 and 1.0, respectively. In Figure 7, the movement path of mobile robot is presented from source to target position. As it can be seen, the fuzzy logic-based redirecting system can appropriately redirect mobile robot to target position without encountering the left, right, and direct obstacles. 
In other word, the automatic redirection of robot can be achieved by correct setting of output membership functions center and using accurate fuzzy rules base which describes the robot reactive behavior in movement to target in presence of obstacles. According to the Figure 8, the robot distance decreases by increasing the repetitions, therefore, it reaches to zero after about 5000 repetitions. Also, in Figure 9 the tracking error is reached to zero in $x$ and $y$ directions after 50 seconds. According to the Figure 10 the linear velocity decreases constantly and robot rotational velocity has larger variance range in some moments.

In the next experiment, it is assumed that target situation is $(2,6)$. The correction coefficient values of orientation membership functions center and linear velocity are considered 1.3 and 1.0, respectively. In Figure 11, the movement path of mobile robot is observed. It can be seen

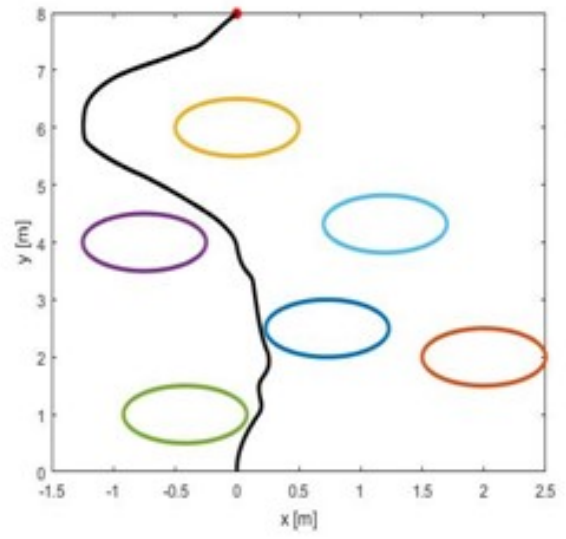

Figure 7. Movement path of mobile robot to second target by using the fuzzy logic.
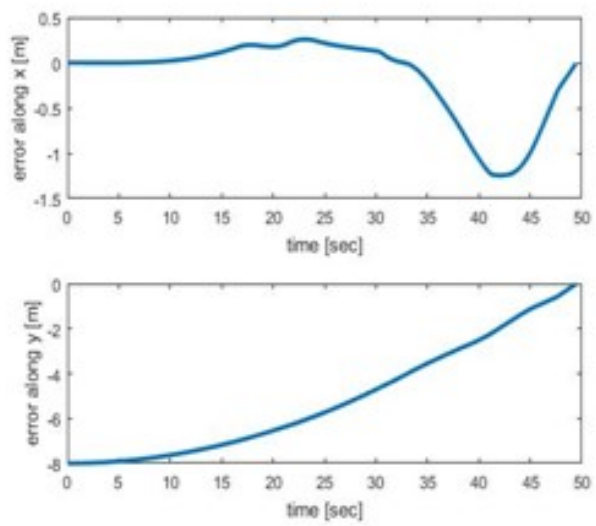

Figure 9. Tracking error of mobile robot in direction of $X$ and $Y$ in tracking of second target position.

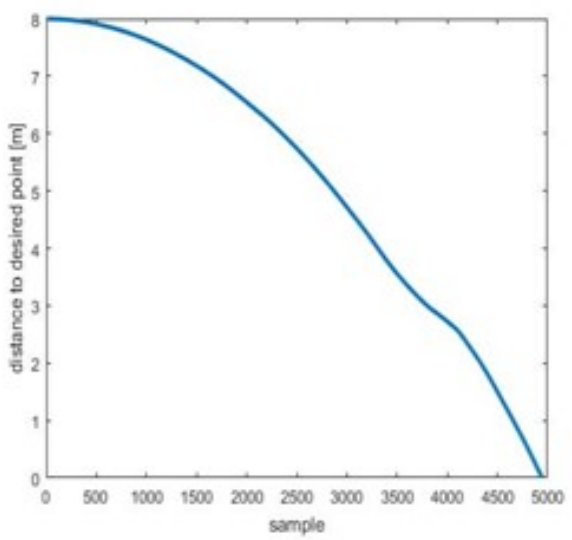

Figure 8. Instantaneous distance of mobile robot from second target position.
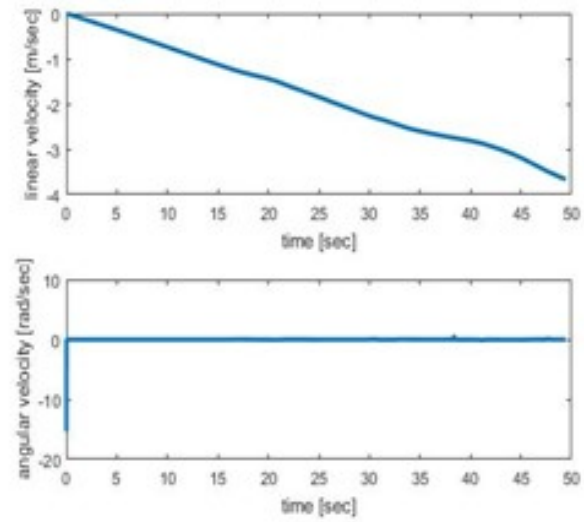

Figure 10. Linear and angular velocity of mobile robot in tracking of second target position.

that the mobile robot reached to the third target without encountering any obstacles. Similar to the two first targets, in this case, the fuzzy logic based redirecting is appropriately performed. 
Figures 12 and 13 indicate the accuracy of tracking target position using proposed redirecting system. It can be observed from Figure 12 that the robot distance decreases and it reaches to zero at about 6500. Figure 13 shows the tracking error of mobile robot in direction of $X$ and $Y$ in tracking of third target position. In direction of $X$ the error is almost steady until 50 seconds and then the error increases. However, in the direction of $Y$ the error is increased after 10 seconds. Also, it can be understood from Figure 14 that linear velocity similar to the two primary targets has an appropriate uniformity and decreasing behavior. Unlike the first two targets, the rotational speed does not have much variance range.

\subsection{Assessing the Redirecting System of Robot Based on Fuzzy Logic by Combining Localization}

The purpose of localization of the mobile robot is to estimate the position and instantaneous orientation of the mobile robot in spite of presence of noises of process and measurement. For

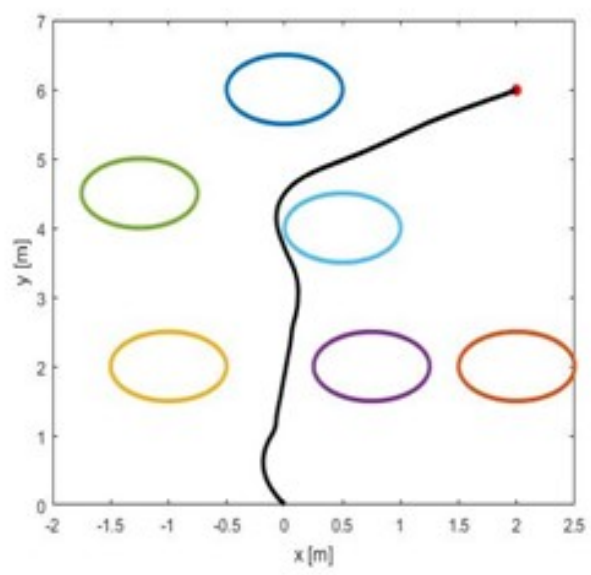

Figure 11. Movement path of mobile third target by using the fuzzy logic
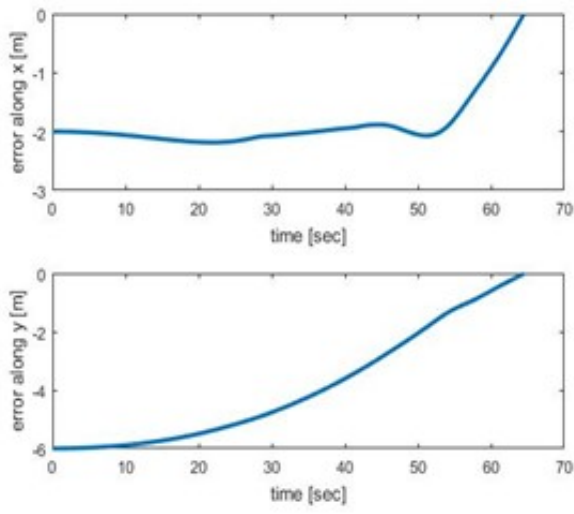

Figure 13. Tracking error of mobile robot in direction $X$ and $Y$ in tracking of third target position

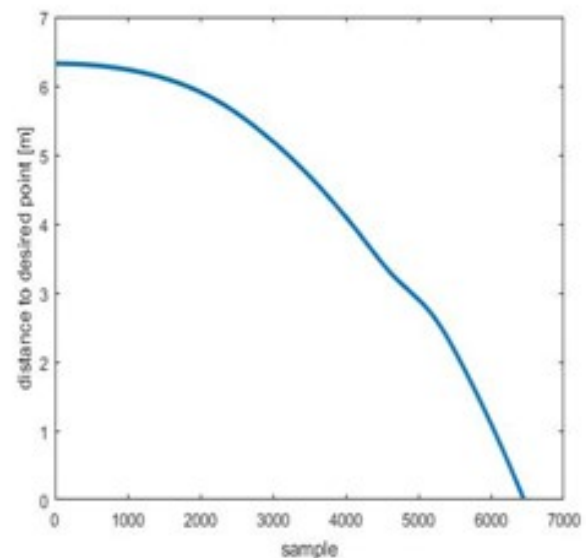

Figure 12. Instantaneous distance of mobile robot to robot from third target position.
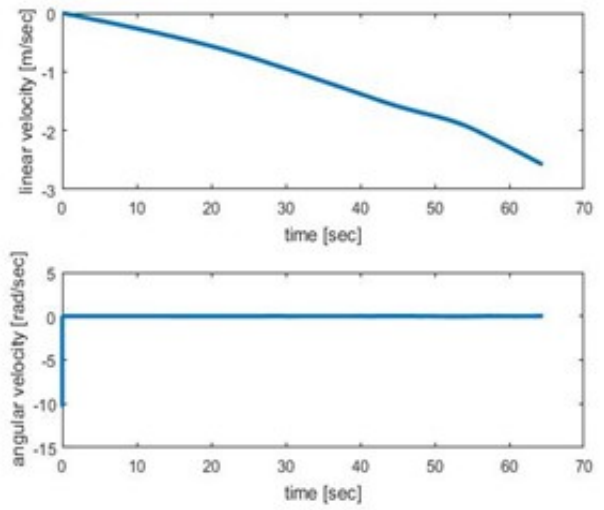

Figure 14. Linear and angular velocity of mobile of robot in tracking of third target position. 
this purpose, the extended Kalman filter was used. The reason for using the Kalman filter is that it gives the best possible estimate despite the presence of noise. In this section, the Kalman filterbased localization problem is combined with fuzzy logic-based redirecting. In other words, the robot's distance is determined from the left, right and direct obstacles, as well as the target orientation in each moment based on the Kalman filter estimation. In Figure 15, a block diagram of redirecting system and a closed loop localization has been presented. For localization of mobile robot using Kalman filter, it is assumed that noises of process and measurement have Gaussian nature with variance 1 . Before combining fuzzy logic-based redirecting with the Kalman filter, the robot performance is investigated in routing without using Kalman filter by applying the noise with variance 0.1 . Figure 16 shows that the robot has encountered difficulties in its routing, even though the stop status has been reduced from 0.01 to 0.1 . It is also observed that the performance of the fuzzy system has encountered difficulties in the presence of noise, and the robot have problem to find its path. Figure 17 shows the instantaneous distance of the mobile robot from first target position. It is noted that the robot reaches to its target after 10000 samples. However, in Figure 18, the movement path of the

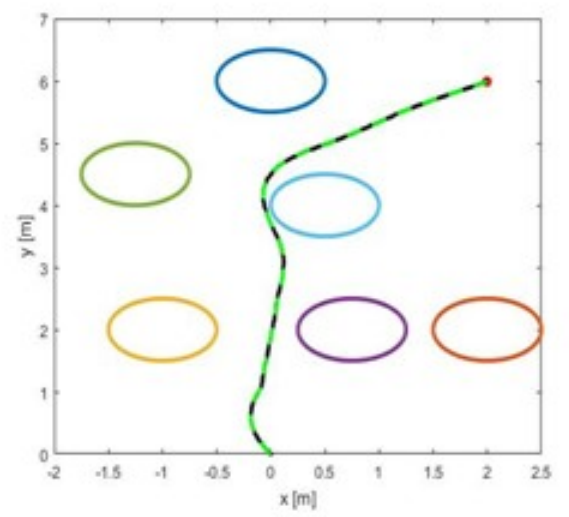

Figure 15. The closed loop structure of redirection localization based on Fuzzy logic and extended Kalman filter.

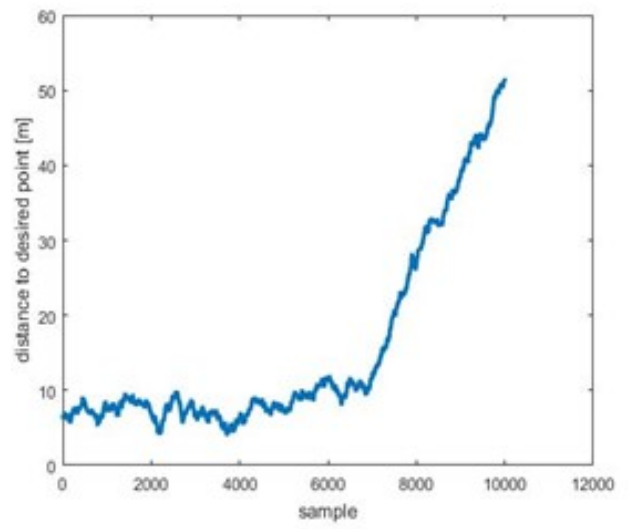

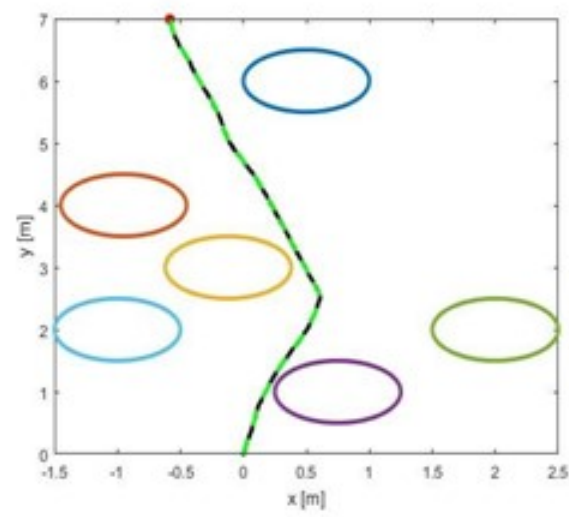

Figure 16. Movement path of the mobile and robot toward the first target by using the fuzzy logic in the presence of noise

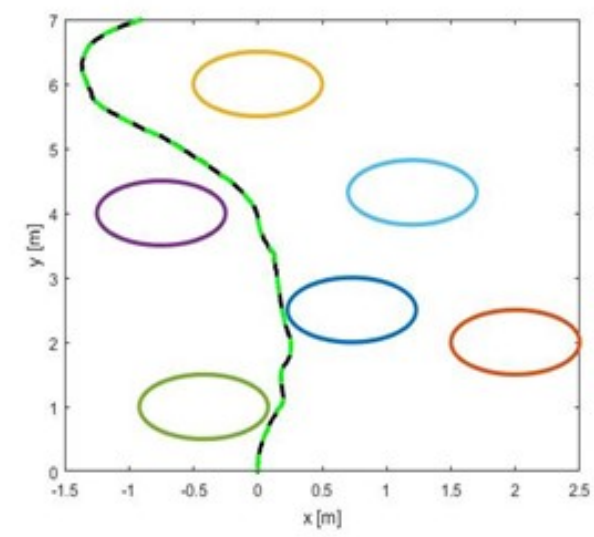

Figure 17. Instantaneous distance of the mobile robot from first target position.
Figure 18. movement path of the mobile robot toward $(0,8)$ target by using the fuzzy logic and extended Kalman filter. 


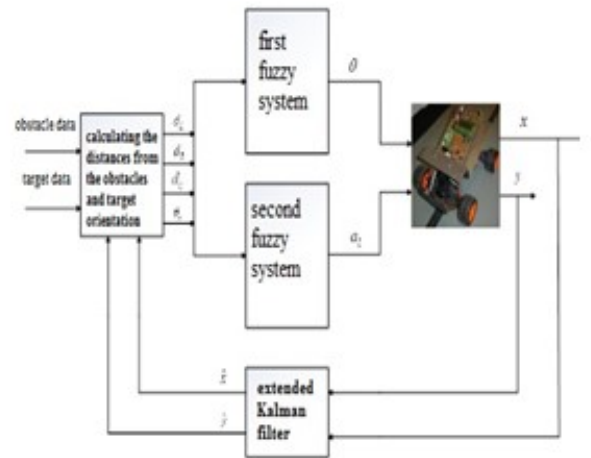

Figure 15. The closed loop structure of redirection localization based on Fuzzy logic and extended Kalman filter.

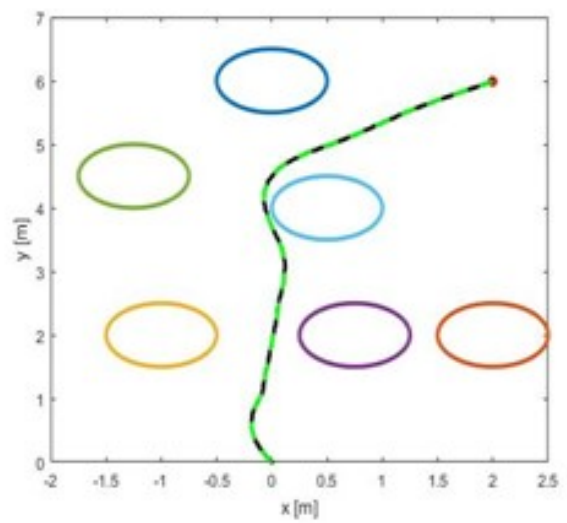

Figure 17. Instantaneous distance of the mobile robot from first target position

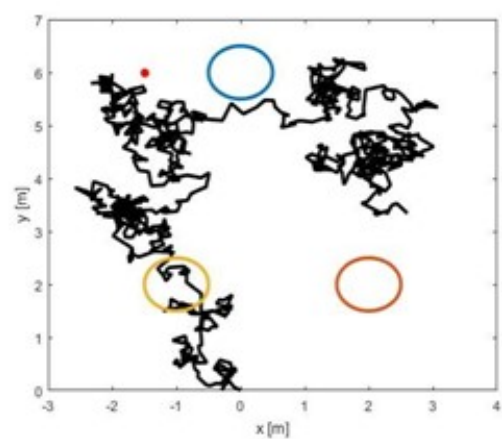

Figure 16. Movement path of the mobile robot and toward the first target by using the fuzzy logic in the presence of noise

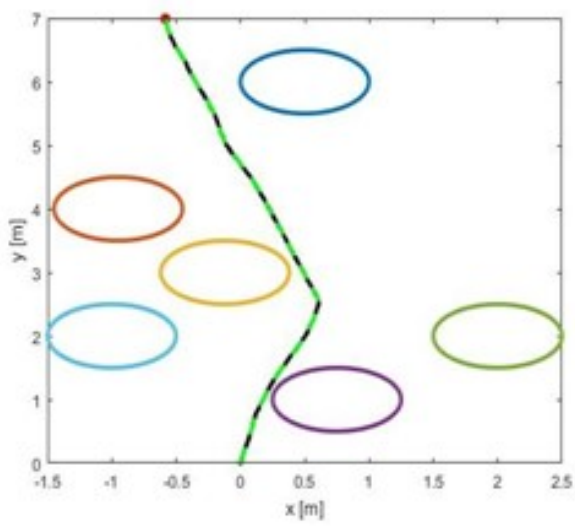

mobile robot has been shown in the presence of noise using extended Kalman filter. As it can be seen, the mobile robot moves appropriately on its movement path to the target position. Note that this appropriate redirecting is performed in the presence of noises of process and measurement with variance 1 . The distance approaches to zero between robot and the target over the time and for most moments, the state estimation error is less than $5 \%$.

Our final experiments have been shown in Figures 19 and 20 for the target position $(2,6)$ and ($0.5,7)$. As it is shown in Figure 19 , the target position of $(2,6)$ has been tracked appropriately without avoiding the obstacle. Also, the distance reaches to zero between the robot and the target over the time and the status of error is approximated less than $1 \%$ using the extended Kalman filter. Therefore, in general, it can be expressed that the redirecting and localization algorithm based on the fuzzy logic and extended Kalman filter works well for estimating the optimum state of the system in the presence of noise. It also works well to redirect accurately the robot toward the target position, and to avoid collision with obstacles. Also, the proposed algorithm is able to track different target positions in the robot's workspace. To illustrate the high quality of the proposed method, the simulation result presented in Figure 20 for a different target position such 
International Journal of Fuzzy Logic Systems (IJFLS) Vol.8, No.3, July 2018.

as $(-0.5,7)$ shows that the robot converges to the target in the presence of noises of process and measurements and obstacles in the robot's work space.

\section{CONCLUSION}

The proposed structure consists of two main subsystems: localization based on the extended Kalman filter and fuzzy logic based redirecting. In order to form an automated redirecting system, it is assumed that circular obstacles are located with determined position in the robot's workspace. Also, the target position is specified in the space. The purpose of the controlling system is to accurately redirect the robot from source toward target position without collision with obstacles. For this purpose, two fuzzy systems were considered. The input components of both systems are the obstacle distance from the center of the left, right, and direct obstacles of the mobile robot and the target orientation. Based on the information received from these components and the formation of a suitable fuzzy rule base, the instantaneous orientation of the robot is determined by the first fuzzy system and the linear acceleration of the mobile robot by the second fuzzy system. The fuzzy rules base consists of forty-two bases which are extracted from the distance of the robot to obstacles, and also the target position relative to the instantaneous orientation of the robot. Also, the single fuzzifier, center average defuzzification, and minimal inference engine have been considered in the structure of fuzzy systems. On the other hand, the extended Kalman filter localization has been used because the instantaneous information of the position of the mobile robot is corrupted by noise. The purpose of localization is the instantaneous estimation of the robot's position in the presence of noises of process and measurement. Accordingly, the input components of the fuzzy systems have been determined based on the estimation of the localization process and the information of the obstacle center and the target position. Finally, the linear acceleration and instantaneous orientation of the mobile robot are determined using the desired fuzzy structures and these apply to its kinematic model. Also, in order to improve the performance of the proposed algorithm for redirecting and positioning mobile robot, the following strategies and issues will be recommended: in this study, the correction factor was used for the center's membership functions of fuzzy system outputs to achieve the correct redirect of the robot from the origin toward the various positions of the purpose. The use of adaptive algorithms for a momentary updating the centers can cause high generalizability of the proposed algorithm. According to the presented results, the proposed algorithm can only be used for the purpose of accurate redirecting without collisions with obstacles. At same time the extra energy will be applied to the robot at some of the paths because the path is longer than expected. Therefore, using an appropriate algorithm which could consider the shortest path in the fuzzy logic application is the significant development in order to improve proposed algorithm.

\section{REFERENCES}

[1] A.V. Topalov. (2011). Recent Advances in Mobile Robots. Rijeka, Croatia: InTech.

[2] S. Y. Chen. (2012). Kalman Filter for Robot Vision: A Survey. IEEE Transactions on Industrial Electronics, 59, 4409-4420.

[3] H. Hur and H. S. Ahn. (2013). Discrete-Time Ho Filtering for Mobile Robot Localization Using Wireless Sensor Network. IEEE Sensors Journal, 13, 245-252.

[4] M. Mirkhani, R. Forsati, A. M. Shahri, and A. Moayedikia. (2013). A Novel Efficient Algorithm for Mobile Robot Localization. Robotics and Autonomous Systems, 61, 920-931.

[5] E. DiGiampaolo, and F. Martinelli. (2014). Mobile Robot Localization Using the Phase of Passive UHF RFID Signals. IEEE Transactions on Industrial Electronics, 61, 365-376.

[6] H. Kim, T. Oh, D. Lee, M. Chung, and H. Myung. (2013). Mobile Robot Localization by Matching 2D Features to 3D Point Cloud. 10th International Conference on Ubiqutous Robots and Ambient Intelligence, Jeju, Korea, pp 266-267. DOI 10.1109/URAI.2013.6677364 
International Journal of Fuzzy Logic Systems (IJFLS) Vol.8, No.3, July 2018.

[7] H. Zhang, J. Chen, and K. Zhang. (2013). Reliable and Efficient RFID-Based Localization for Mobile Robot. IEEE International Symposium on Robotic and Sensors Environment, Washington, DC, USA, pp 184-189. DOI 10.1109/ROSE.2013.6698440

[8] E. Colle, and S. Galerne. (2013). Mobile Robot Localization by Multiangulation Using Set Inversion. Robotics and Autonomous Systems, 61, 39-48.

[9] T. Thanh, V. Nguyen, M. D. Phung, T. H. Tran, and Q. V. Tran. (2012). Mobile Robot Localization Using Fuzzy Neural Network Based Extended Kalman Filter. 2012 IEEE International Conference on Control System, Computing and Engineering, Penang, Malaysia, pp 23-25. DOI 10.1109/ICCSCE.2012.6487181

[10] G. G. Rigatos. (2010). Extended Kalman and Particle Filtering for Sensor Fusion in Motion Control of Mobile Robots. Mathematics and Computers in Simulation, 81, 590-607.

[11]H. H. Lin, C. C. Tsai, and J. C. Hsu. (2008). Ultrasonic Localization and Pose Tracking of an Autonomous Mobile Robot via Fuzzy Adaptive Extended Information Filtering. IEEE transactions on Instrumentation and Measurement, 57, 2024-2034.

[12] M. Begum, G. K.I. Mann, and R. G. Gosine. (2008). Integrated Fuzzy Logic and Genetic Algorithmic Approach for Simultaneous Localization and Mapping of Mobile Robots. Applied Soft Computing, 8, $150-165$.

[13]H. Najjaran and A. Goldenberg. (2007). Real-Time Motion Planning of an Autonomous Mobile Manipulator Using a Fuzzy Adaptive Kalman Filter. Robotics and Autonomous Systems, 55, 96-106.

[14]K. Demirli and M. Molhim. (2004). Fuzzy Dynamic Localization for Mobile Robots. Fuzzy Sets and Systems, 144, 251-283.

[15] L. D’Alfonso, A. Griffo, P. Muraca, and P. Pugliese. (2013). A SLAM Algorithm for Indoor Mobile Robot Localization Using an Extended Kalman Filter and a Segment Based Environment Mapping. 16th IEEE International Conference on Advanced Robotic, Montevideo, Uruguay, pp 1-6. DOI 10.1109/ICAR.2013.6766461

[16] M. Pinto, A. P. Moreira, and A. Matos. (2012). Localization of Mobile Robots Using an Extended Kalman Filter in a LEGO NXT. IEEE Transactions on Education, 55, 135-144.

[17] C. J. WU and C. C. Tsai. (2001). Localization of an Autonomous Mobile Robot Based on Ultrasonic Sensory Information. Journal of Intelligent and Robotic Systems, 30, 267-277.

[18] J. J. Leonard and H. F. D. Whyte. (1991). Mobile Robot Localization by Tracking Geometric Beacons. IEEE transactions on Robotics and Automations, 1, 376-382.

[19] L. Jetto, S. Longhi1, D. Vitali. (1999). Localization of a Wheeled Mobile Robot by Sensor Data Fusion Based on a Fuzzy Logic Adapted Kalman Filter. Control Engineering Practice, 7, 763-771.

[20] Q. h. Meng, Y. c. Sun, and Z. 1. Cao. (2000). Adaptive Extended Kalman Filter (AEKF)-Based Mobile Robot Localization Using Sonar. Robotica, 18, 459-473.

[21] G. Alefeld and J. Herzberger. (1984). Introduction to Interval Computation (pp.210-225). New York, USA: Academic press.

[22] I. Arasaratnam and S. Haykin. (2008). Square-root Quadrature Kalman Filtering. IEEE Transactions on Signal Processing, 56, 2589-2593. 